\title{
ANTROPOMETRIA E SOMATOTIPO: \\ FATORES DETERMINANTES NA SELEÇÃO DE ATLETAS NO VOLEIBOL BRASILEIRO
}

\author{
MS. BRENO GUILHERME DE ARAUJO T. CABRAL \\ Mestre em Ciências da Saúde pela Universidade Federal do Rio Grande do Norte \\ Professor assistente do Departamento de Educação Física da Universidade Federal do \\ Rio Grande do Norte (Natal - Rio Grande do Norte - Brasil) \\ e-mail: brenotcabral@hotmail.com
}

DRA. SUZET DE ARAUJO TINÔCO CABRAL

Doutora em Ciências da Saúde pela Universidade Federal do Rio Grande do Norte Professora Associada I do Departamento de Educação Física da Universidade Federal do

Rio Grande do Norte (Natal - Rio Grande do Norte - Brasil) e-mail: suzet@ufrnet.br

\author{
ESP. ISABELLA VIANNA R. G. TOLEDO \\ Especialista em nutrição esportiva \\ Nutricionista da Confederação Brasileira de Voleibol \\ (Rio de Janeiro - Rio de Janeiro - Brasil) \\ e-mail: isabellatoledo@globo.com
}

DR. PAULO MOREIRA SILVA DANTAS

Doutor em Ciências da Saúde pela Universidade Federal do Rio Grande do Norte Professor adjunto II do Departamento de Educação Física da Universidade Federal do Rio Grande do Norte (Natal - Rio Grande do Norte - Brasil) e-mail:pgdantas@ufrnet.br

\section{DR. HÊNIO FERREIRA DE MIRANDA}

Doutor em Ciências da Saúde pela Universidade Federal do Rio Grande do Norte (Brasil) Professor Associado II do Departamento de Educação Física da Universidade Federal do Rio Grande do Norte (Natal - Rio Grande do Norte - Brasil) e-mail: henio@ccs.ufrn.br

\section{DRA. MARIA IRANY KNAKCFUSS}

Doutora em Ciências da Saúde pela Universidade Federal do Rio Grande do Norte Professor adjunto do Departamento de Educação Física da Universidade Estadual Regional do

Rio Grande do Norte (Natal - Rio Grande do Norte - Brasil) e-mail:mik@ufrnet.br 


\section{RESUMO}

Objetivo: Analisar a antropometria e somatotipo de atletas do voleibol brasileiro. Metodologia: a amostra foi composta por 54 atletas convocadas para as seleções nacionais, sendo

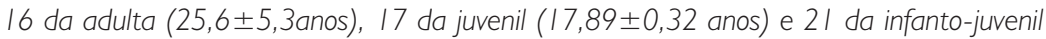
(15,86 $\pm 0,36$ anos). Para o somatotipo utilizou-se o método de Heath e Carter. O tratamento estatístico utilizado foi o descritivo com valores de tendência central e seus derivados, e o inferencial ANOVA e post hoc de Tukey. Para o somatotipo recorreu-se ao cálculo da distância espacial entre os somatótipos (DES). Resultados: A variável estatura não apresenta diferença significativa entre as seleções, somente entre as posições de jogo. O somatotipo da seleção infanto-juvenil foi diferente das demais. A seleção adulta classificou-se como ectomorfo-mesomorfo $(2,24 / 3,16 / 3,58)$, juvenil como central $(3,12 / 3,40 / 3,30)$ e infantojuvenil como ecto-endomorfico $(3,07 / 2,25 / 3,81)$. Conclusão: a estatura e linearidade são variáveis importantes na seleção de atletas para o alto rendimento.

PALAVRAS-CHAVE: Antropometria; somatotipo; voleibol; performance.

\section{INTRODUÇÃO}

sucesso de uma modalidade esportiva em campeonatos mundiais representa grande incentivo para a população de um país, despertando interesse pela prática esportiva da modalidade em foco (OKAZAK et al. , 20 I I). O voleibol é o segundo esporte mais praticado no Brasil e nos últimos dez anos conquistou a condição de recordista mundial de títulos nas categorias de base, tornou-se bi-campeão olímpico no masculino e campeão olímpico no feminino, exigindo um nível cada vez melhor da condição física dos atletas praticantes dessa modalidade (CBV, 2009; FLORES et al., 2009).

A evolução do desporto moderno conduziu os pesquisadores das Ciências do Desporto a investigarem os aspectos fundamentais da excelência do alto rendimento. A identificação de aspectos físicos é de grande relevância para os treinadores, uma vez que, o voleibol requer características físicas específicas para cada posição de jogo. Estudos tem apontado a necessidade de se obter o tipo físico ideal em cada posição de jogo, para facilitar os programas de treinamento de acordo com a demanda técnica e fisiológica exigida durante o jogo em cada posição. Observa-se ainda que, a habilidade técnica, analisada de forma isolada, não é suficiente para se alcançar o alto nível no voleibol mundial, o qual exige padrões das características antropométricas, fisiológicas e neuromusculares específicas a modalidade (MALOUSARIS et al., 2008; DUNCAN; WOODFIELD; AL-NAKEEB, 2006).

A busca de variáveis que identifiquem o futuro atleta ainda na iniciação tem sido um desafio para os pesquisadores, pois sabemos que a técnica pode ser adquirida 
com o treinamento de qualidade, entretanto, em estudos especificos com voleibol, a literatura aponta que para se alcançar o alto rendimento passa a ser necessário à observação de variáveis antropométricas como estatura e somatotipo aliadas as habilidades e treinamento (MALOUSARIS et al., 2008; CABRAL et al., 201 I; MALINA; BOUCHARD; BAR-OR, 2004).

Observa-se que, com a evolução do esporte que houveram mudanças das características antropométricas do atleta comparando-se os estudos de Vivolo, Caldeira e Matsudo (1980), onde a média de estatura da seleção adulta do Brasil era $174,29 \pm 3,91 \mathrm{~cm}$, com os dados do presente estudo na mesma categoria $(|82,8| \pm 7,0 \mathrm{~cm})$.

As características antropométricas e da composição corporal são cada vez mais decisivas na seleção e determinação de rendimento dos atletas, proporcionando melhores condições para o treino das qualidades físicas, além de auxiliarem diretamente nas ações de jogo (NORTON; OLDS, 200I).

A literatura internacional cita a importância da somatotipologia no esporte em pesquisa realizada com atletas do sexo feminino na Itália no qual demonstram a homogeneidade do somatotipo no referido nível de qualificação, mas foi observado que dentro do grupo o somatotipo difere de acordo com as posições de jogo (GUALDI-RUSSO; ZACCAGNI, 200I).

Massa et al. (2000) comentam ainda, que o somatotipo vem sendo utilizado na comunidade científica para conhecer-se o tipo físico ideal para cada modalidade esportiva, sendo assim, um excelente método de auxílio na orientação esportiva e descoberta de talentos. Os mesmos autores em estudos com diferentes categorias no voleibol feminino, concluíram que o componente endomórfico predomina nas categorias inferiores e vai diminuindo com o processo maturacional, à medida que aumenta de categoria até que passa a ser o terceiro componente na categoria adulta, provavelmente ocasionado por maiores exigências no treinamento e nas competições, corroborando assim os estudos de Veiga (2009) com atletas de handebol, que cita a influencia da maturação e treinamento nas características somatotipicas.

Observa-se na literatura atual o uso do somatotipo para identificar as características específicas nas diversas modalidades esportivas, assim como as características nas diferentes posições de jogo com estudos comparativos relevantes no basketball, handball e volleyball (BAYOS et al. 2006); soccer e handball (RASCHKA; WOLTHAUSEN, 2007); carate (FRITZSCHE; RASCHKA, 2007) e tênis (SÁNCHEZMUNOZ; SANZ; ZABALA, 2007). 
Diante do exposto, considerando que as informações a respeito da antropometria e somatotipo em atletas de elite são relevantes para a ciência do esporte, permitindo estabelecer comparações com as equipes mais jovens e auxiliando na busca de novos atletas para o alto rendimento, e observando a necessidade de avaliação de tais características como fatores determinantes para se alcançar o alto rendimento em modalidades como o voleibol, na qual o Brasil apresenta excelentes resultados sendo recordista mundial de títulos nas diferentes categorias, é que, a presente investigação teve como propósito classificar e comparar o somatotipo nas diferentes categorias competitivas e posições de jogo da elite do voleibol feminino brasileiro.

\section{METODOS}

\section{SUJEITOS}

A amostra foi composta por cinquenta e quatro atletas do sexo feminino convocadas para as seleções brasileiras para representar o país em competições internacionais em 2007, 2008 e anos subsequentes nas categorias: infanto-juvenil $(n=21)$ com idade entre 14 e 16 anos, juvenil $(n=17)$ com idade entre 15 e 18 anos e adulta $(n=16)$ sem limite de idade. A coleta de dados foi realizada no centro de treinamento de Saquarema no Rio de Janeiro/Brasil, no período básico do treinamento centralizado. A investigação seguiu rigorosamente os protocolos escolhidos para observação de antropometria e composição corporal, respeitando as normas de ética em pesquisa, onde os procedimentos realizados foram previamente autorizados pelo Comitê de ética da Universidade Federal do Rio Grande do Norte (Parecer n. I 19/07), atendendo aos itens propostos pela resolução 196/96-CNSBrasil, assim como respeitando as normas internacionais de experimentação com humanos (DECLARAÇÃO DE HELSÍNKI DE 1975).

\section{PROCEDIMENTOS PARA AS CARACTERISTICAS ANTROPOMÉTRICAS E SOMATOTÍPICAS}

As medidas antropométricas foram tomadas de acordo com o manual de ISAK (MARFELL-JONES et al., 200 I ) verificando a estatura e massa corporal através de uma balança antropométrica mecância (Filizola, Brasil) com precisão de 100 gramas e estadiômetro (Sanny, Brasil) com precisão de 0, I cm. Para o somatotitpo foi utilizado o método somatotipológico de Heath e Carter (1967). As medidas de dobras cutâneas foram realizadas com um compasso de dobras cutâneas Langer (Cambridge, Maryland) em quatro locais (tríceps, subescapular, suprailíaca e medial de perna) próximo de 0, I mm, as medidas foram realizadas de acordo com a técnica de Carter e Heath (1990). A circunferência do braço (cm) foi medida em contração e a 
da perna $(\mathrm{cm})$ foi medida com o sujeito em pé utilizando uma trena antropométrica de metal flexível (Sanny, Brasil) com $2 \mathrm{~m}$ de comprimento e precisão de $0,1 \mathrm{~cm}$. Os diâmetros umeral e femural foram tomados com um paquímetro (Sanny, Brasil) cuja variação fica entre 02 e $15 \mathrm{~cm}$ e com graduação é de 0,05 mm.

Todas as medidas foram realizadas do lado direito do corpo seguindo padrões unificados, em um ambiente fechado e no mesmo horário de avaliação, pelo mesmo avaliador, com os sujeitos usando roupas leves, sem calçados.

\section{TRATAMENTO ESTATÍSTICO}

Utilizou-se a estatística descritiva para se obter a média e o desvio padrão, e a estatística inferencial para comparação dos dados através do teste Anova one-way e post hoc de Tukey, contidos no pacote estatístico SPSS versão I6, considerandose o nível de significância $p<0.05$. O teste de Kolmogorov-Smirnov demonstrou a normalidade dos dados. As comparações foram realizadas para o teste inter-grupos nas três categorias e intra-grupos nas diferentes posições de jogo.

Com o intuito de analisar os dados do somatotipo das atletas, recorreu-se ao cálculo da distância espacial entre os somatótipos (DES) (MARFELL-JONES et al., 200 I; DUQUET et al., 1977). Esta técnica consiste na medida da distância no espaço tridimensional entre dois somatotipos ( $\mathrm{A}$ e $\mathrm{B}$ ) que, em termos práticos, leva em consideração a amplitude entre cada somatotipo com um somatotipo de referência. Considera-se como variação significativa entre os somatotipos valor da DES igual ou maior a uma unidade. A dispersão do somatotipo de I,0 é equivalente à mudança de uma unidade na classificação de um componente. Este valor $(1,0)$ é usado para indicar diferenças entre somatotipos médios de atletas. A distância é o resultado da raiz quadrada da soma dos quadrados das diferenças entre cada componente, obtida pela seguinte fórmula:

$$
D E S=\sqrt{ }\left(I_{A}-I_{B}\right)^{2}+\left(I I_{A}-\| I_{B}\right)^{2}+\left(I I I_{A}-\| I_{B}\right)^{2}
$$

Na qual: DES = distância espacial entre os somatótipos; I= endomorfia; $\|=$ mesomorfia; $\| I=$ ectomorfia; $A$ e $B=$ índice de dois somatótipos a serem comparados.

\section{RESULTADOS}

A Tabela I descreve os resultados por categoria para as variáveis: estatura, massa corporal, endomorfia, mesomorfia e ectomorfia. Quanto à classificação a 
seleção adulta, foi considerada como ectomorfo-mesomorfo, a seleção juvenil como central e a seleção infanto-juvenil como endo-ectomorfo.

Tabela I. Descrição por categoria quanto a estatura, massa corporal, endomorfia, mesomorfia e ectomorfia.

\begin{tabular}{lllllll}
\hline Categoria & & Estatura $\mathrm{cm}$ & Massa kg & Endo & Meso & Ecto \\
\hline Seleção adulta & $\mathrm{N}$ & 16 & 16 & 16 & 16 & 16 \\
\hline & Média & 182,81 & 72,56 & 2,24 & 3,16 & 3,58 \\
\hline & $\mathrm{DP}$ & 7,00 & 6,44 & 0,59 & 1,08 & 1,03 \\
\hline Seleção juvenil & $\mathrm{N}$ & 17 & 17 & 17 & 17 & 17 \\
\hline & Média & 181,66 & 73,36 & 3,12 & 3,40 & 3,30 \\
\hline & $\mathrm{DP}$ & 7,16 & 9,40 & 0,57 & 0,89 & 0,83 \\
\hline Seleção infanto-juvenil & $\mathrm{N}$ & 21 & 21 & 21 & 21 & 21 \\
\hline & Média & 181,63 & 68,11 & 3,07 & 2,25 & 3,81 \\
\hline & $\mathrm{DP}$ & 6,12 & 8,72 & 0,84 & 1,07 & 1,29 \\
\hline
\end{tabular}

Nível de significância adotado para comparar a estatura e massa corporal $p<0,05$.

Os resultados do ANOVA e post hoc Tukey mostraram não haver diferença significativa entre as seleções para estatura e massa corporal. Na comparação do somatótipo entre as seleções, considerando o DES $\geq 1,00$, os resultados mostram diferença na distância espacial entre os somatótipos da seleção infanto-juvenil com as demais seleções, observados os seguintes valores: adultas $\times$ infanto-juvenis (DES= $1,25)$ e juvenis $x$ infanto-juvenis (DES $=1,25)$.

Tabela 2. Descrição por posição de jogo quanto à estatura,

massa corporal, endomorfia, mesomorfia e ectomorfia.

\begin{tabular}{|c|c|c|c|c|c|c|}
\hline \multicolumn{2}{|l|}{ Categoria } & \multirow{2}{*}{$\begin{array}{l}\text { Estatura }(\mathrm{cm}) \\
7 \\
171,00^{\mathrm{a}} \\
3,69\end{array}$} & \multirow{2}{*}{$\begin{array}{l}\text { Massa }(\mathrm{kg}) \\
7 \\
61,65^{c} \\
5,11\end{array}$} & \multirow{2}{*}{$\begin{array}{l}\text { Endo } \\
7 \\
2,57 \\
0,62\end{array}$} & \multirow{2}{*}{$\begin{array}{l}\text { Meso } \\
7 \\
3,48 \\
071\end{array}$} & \multirow{2}{*}{$\begin{array}{l}\text { Ecto } \\
7 \\
3,16 \\
0,38\end{array}$} \\
\hline Líbero & $\begin{array}{l}\mathrm{N} \\
\text { Média } \\
\mathrm{DP}\end{array}$ & & & & & \\
\hline Levantadora & $\mathrm{N}$ & 9 & 9 & 9 & 9 & 9 \\
\hline & Média & $178,20^{b}$ & 69,36 & 3,36 & 3,35 & 3,23 \\
\hline & DP & 4,31 & 5,05 & 0,58 & 0,83 & 0,68 \\
\hline Central & $\begin{array}{l}\mathrm{N} \\
\text { Média } \\
\text { DP }\end{array}$ & $\begin{array}{l}15 \\
187,44 \\
4,59\end{array}$ & $\begin{array}{l}15 \\
75,28 \\
8,57\end{array}$ & $\begin{array}{l}15 \\
2,66 \\
0,90\end{array}$ & $\begin{array}{l}15 \\
2,42 \\
0,85\end{array}$ & $\begin{array}{l}15 \\
3,72 \\
1,33\end{array}$ \\
\hline Ponta & $\begin{array}{l}N \\
\text { Média } \\
\text { DP }\end{array}$ & $\begin{array}{l}14 \\
183,55 \\
4,37\end{array}$ & $\begin{array}{l}\mid 4 \\
70,75 \\
8,4 \mid\end{array}$ & $\begin{array}{l}14 \\
2,85 \\
0,81\end{array}$ & $\begin{array}{l}14 \\
2,70 \\
1,5 \mid\end{array}$ & $\begin{array}{l}14 \\
3,95 \\
1,17\end{array}$ \\
\hline Oposta & $\begin{array}{l}\text { N } \\
\text { Média } \\
\text { DP }\end{array}$ & $\begin{array}{l}9 \\
182,75 \\
3,02\end{array}$ & $\begin{array}{l}9 \\
74,05 \\
8,47\end{array}$ & $\begin{array}{l}9 \\
2,80 \\
0,75\end{array}$ & $\begin{array}{l}9 \\
3,06 \\
1,09\end{array}$ & $\begin{array}{l}9 \\
3,38 \\
1,14\end{array}$ \\
\hline
\end{tabular}

Nível de significância adotado para comparar a estatura e massa corporal $p<0,05$.

$a=$ líbero $\neq$ levantadora, central, ponta e oposta; $b=$ levantadora $\neq$ central e ponta;

$c=$ líbero $\neq$ levantadora, central, ponta e oposta. 
$\mathrm{Na}$ Tabela 2, onde os grupos foram separados por posição de jogo, constatouse existir diferença quanto à antropometria e somatotipia em algumas posições, ou seja, parece existir um perfil físico específico por posição de jogo no alto rendimento, independente da categoria. Observa-se ainda quanto aos componentes do somatotipo, que a ectomorfia, que está relacionada à linearidade, apresenta valores acima de 3,0 em todas as posições de jogo, demonstrando sua importância na seleção de atletas para o alto rendimento. Ficando a seguinte classificação somatotipica para as posições: Libero (Mesomorfo-ectomorfo), Levantadora (Central), Central e Ponta (Ectomorfa balanceada) e Oposta (Ectomorfo-mesomorfo).

Após a aplicação do ANOVA One-way e Post Hoc de Tukey, observando o nível de significância de $\mathrm{p}<0,05$ para as afirmativas da existência de diferença, observa-se os seguintes resultados entre as posições de jogo para estatura: Líbero $x$ Levantadora $(p=0,0$ I I ); Libero x Ponta, Líbero $\times$ Central e Libero $\times$ Oposto $(p<0,00$ I); Levantador $\times$ Central $(p<0,001)$; Levantador $\times$ Ponta $(p=0,030)$. Massa corporal: Libero $\times$ Central $(p=0,003)$ e Libero $\times$ Oposto $(p=0,024)$. Na comparação do somatótipo entre as posições de jogo os resultados mostram diferença na distância espacial entre os somatótipos, considerando o DES $\geq 1,00$, de Libero $\times$ Central $(D E S=1,21)$, Libero $\times$ Ponta $(D E S=1,15)$, Levantador $\times$ Central $(D E S=1,26)$ e Levantador $\times$ Ponta $(\mathrm{DES}=1,10)$.

\section{DISCUSSÕES}

No voleibol atual pode-se dizer que a estatura passa a ser um elemento fundamental para o alcance do alto rendimento, junto com variáveis como, capacidades motoras e estratégias de treinamento (GUALDI-RUSSO; ZACCAGNI, 200 I; BAYIOS et al., 2006; BANDYOPADHYAY, 2007; MALOUSARIS et al., 2008; CABRAL et al., 201 I ).

De acordo com os dados divulgados no site da Federação Internacional de Voleibol sobre o campeonato mundial infanto-juvenil de 2009, observou-se que a estatura média das três primeiras seleções foi 183,2cm (Brasil), I 84,6 cm (Servia) e 181,6 cm (Bélgica) (FIVB, 20 I0). Pode-se observar a evolução da estatura no voleibol mundial através de estudos realizados com a seleção brasileira feminina, que na época não tinha expressão internacional, e a seleção japonesa, campeã olímpica e mundial, onde a estatura média era de $174,29 \pm 3,91 \mathrm{~cm}$ e $175,54 \pm 3,31 \mathrm{~cm}$ respectivamente (VIVOLO; CALDEIRA; MATSUDO, 1980) e da equipe adulta do Brasil vice-campeã mundial do Gran Prix em 2010 que obteve uma média de I85,2l $\pm 6,35 \mathrm{~cm}$ (FIVB, 20 I0). 
Malousaris et al. (2008) em estudos com 163 atletas adultas de elite das divisões A e B do campeonato nacional da Grécia encontraram uma média de estatura próxima a de nossas levantadoras $(177,1 \pm 6,5 \mathrm{~cm}$ ), embora quando dividiram 0 grupo por nível de performance, a divisão I obteve 179,6 \pm 5,8cm e a divisão II $174,7 \pm 6,2 \mathrm{~cm}$. A média de estatura encontrada para jogadores de voleibol na Índia, para o sexo masculino foi $173,10 \pm 4,19 \mathrm{~cm}$, inferior a nossa amostra no feminino, no qual o autor considerou grande desvantagem uma vez que essa variável passou a ser fundamental para o sucesso na modalidade (BANDYOPADHYAY, 2007). Gabbett e Georgieff (2007) em estudos com jogadoras de voleibol australiano em diferentes níveis de performance encontraram valores de estatura para equipes nacionais infanto-juvenis $(179,2 \pm 1,0 \mathrm{~cm})$, estaduais $(179,5 \pm 0,6 \mathrm{~cm})$ e iniciantes $(177,0 \pm 0,6 \mathrm{~cm})$ e importantes achados nesse estudo demonstraram que $\mathrm{o}$ alcance de ataque é uma das variáveis determinantes para diferenciar os três níveis de performance. Estudo no Brasil com a seleção estadual feminina do Rio Grande do Norte encontrou uma média de estatura bem abaixo dos citados anteriormente com valores de 169,4 \pm 7,97cm (CABRAL; BARBOSA; CABRAL, 2005).

Observa-se que a estatura varia de acordo com a posição de jogo e que a líbero (atleta específica da defesa) aparece como a jogadora mais baixa em nosso estudo, o que pode ser justificado pela função que requer muita agilidade, tempo de reação e excelente habilidade técnica para os fundamentos da defesa e passe, onde certamente a estatura não tem fundamental importância (BELLENDIER, 2003). No entanto, foi observado um alto componente mesomórfico na referida posição de jogo, o que possivelmente influencia no desenvolvimento com eficiência das características físicas exigidas (BELLENDIER, 2003). As jogadoras de meio ou centrais, foram as de maior estatura, fato justificado pela necessidade de um maior alcance de bloqueio nas três posições de ataque, função da referida posição de jogo para tentar impedir o ataque adversário.

Importante salientar que o estudo ora apresentado demonstra que a simples observação da estatura como critério de determinação da performance, fica enfraquecido se esta informação não for associada as exigências de posição de jogo e a idade, que traz consigo habilidade maior na movimentação, e, que mesmo as posições de maior estatura possuem na necessidade de capacidades de movimentação rápida (agilidade) fundamento importante, corroborando com a literatura (FLORES et al., 2009; OKAZAKI et al., 20 I I ; MARQUES et al., 2009). A partir destas observações fica demonstrado que a linearidade observada pelo somatótipo em muito contribui na sintonia fina destas observações, não sendo a mesma necessariamente ligada a uma alta estatura (MARQUES et al., 2009; MALOUSARIS et al., 2008). 
Quanto ao somatotipo, o nosso estudo corrobora com Massa et al. (2000) quando demonstra que a categoria infanto-juvenil apresenta um valor mais alto de endomorfia em relação a mesomorfia, e que esses valores tendem a inverter, à medida que as atletas mudam de categoria e as exigências físicas aumentam. Tais resultados corroboram estudos de Veiga (2009) que sugere que os valores de mesomorfismo tendem a aumentar a medida que os atletas avançam em seu processo maturacional. Em estudos no voleibol com atletas infanto-juvenis do sexo feminino, participantes de campeonatos estaduais em São Paulo, Massa et al. (2000) encontraram para a categoria os valores: endomorfia $(4,4 \pm 0,9)$, mesomorfia $(2,7 \pm 1,3)$, ectomorfia $(3,7 \pm 1,5)$, sendo a mesma tendência observada no estudo realizado por Cabral et al. (2004) com a equipe feminina infanto-juvenil do Rio Grande do Norte onde os valores médios encontrados foram: endomorfia $(3,9 \pm 1,12)$, mesomorfia $(1,9 \pm 1,19)$ e ectomorfia $(3,2 \pm 1,48)$. Gualdi-Russo e Zaccagni (200 I) observaram o somatotipo em atletas adultas de elite do voleibol

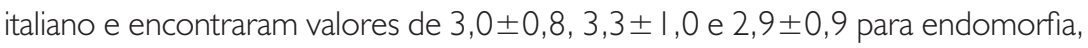
mesomorfia e ectomorfia respectivamente, concluindo que o somatotipo varia de acordo com o nível de performance e com as posições de jogo. Em estudos com I atletas adultas de elite, das divisões A e B do campeonato nacional na Grécia Malousaris et al. (2008) tambem concluiram que as características somatotípicas variam de acordo com o nível de performance.

Em 2006 Bayios e colaboradores, em estudo com 5 I 8 atletas do sexo feminino e de diferentes modalidades esportivas, todas participantes de divisões I e 2 de campeonatos nacionais, observaram que as atletas de voleibol foram as mais altas e a classificação do somatotipo foi endomorfo balanceado $(3,4-2,7-2,9)$. (BAYIOS et al., 2006)

O somatotitpo do presente estudo nas diferentes posições de jogo revelaram um perfil específico para cada função, independente da categoria, onde as atacantes de ponta e de meio foram classificadas como ectomorfa balanceada, as opostas como ectomorfo-mesomorfo, líberos mesomorfo-ectomorfo e as levantadoras enquadradas como centrais, o que difere da classificação encontrada por Malousaris (2008) em atletas gregas da divisão I do campeonato nacional onde as atacantes de ponta, de meio e levantadoras classificaram-se como endomorfo-ectomorfo, as opostas ectomorfas balanceadas e as líberos como centrais.

Duncan, Woodfield e Al-Nakeeb (2006) em estudos com atletas infantojuvenis femininas de alta performance verificaram que as levantadoras apresentam características mais ectomórficas e menos mesomórficas que as centrais com valores para os três componentes: levantadoras $(2,6 \pm 0,9,1,9 \pm 1,1,5,3 \pm 1,2)$ e centrais $(2,2 \pm 0,8,3,9 \pm 1,1,3,6 \pm 0,7)$ o que difere do estudo de Gualdi-Russo 
e Zaccagni (200 I) no qual obteve valores para as levantadoras $(3,1-3,6-2,6)$ e para as centrais $(2,8-3,1-3,1)$, apresentando as centrais um valor para ectomorfia maior que as levantadoras.

Estudos na ciência do esporte tem revelado que as características somatotípicas variam de acordo com a modalidade esportiva, o nível de qualificação e posição de jogo, sendo essa qualificação definida através de resultados em nível internacional (FIVB, 20I0). Quintal et al. (2007) estudando o handebol cita que apesar da relevância da estatura para a modalidade, o componente mesomorfico tem sido considerado um parâmetro de identificação de talentos, enquanto no voleibol Bellendier (2003) afirma que equilíbrio entre os componentes mesomórfico e ectomórfico parece representar a tendência do voleibol mundial, ou seja, atletas fortes, de grande estatura e lineares.

Stamm et al. (2003) concluíram em seus estudos com jogadoras de voleibol, que a estatura é um fator que influencia na performance dos elementos do jogo principalmente (7 I-83\%) nas ações de ataque e bloqueio, sendo assim de grande relevância na seleção de atletas.

A massa critica de estudo sobre a somatotipia no voleibol, repercuti nas seleções nacionais, em estudo recente sobre alcance-ataque, onde Cabral et al. (20 I I) demonstram que, a associação entre variáveis antropométricas e de performance na busca do entendimento do alto rendimento, indica a possibilidade na diminuição do erro nas interpretações dos resultados, quando aparentemente o pouco entendimento na interpretação da somatotipia caracteriza-se como fator limitante a sua utilização.

Em acordo com a literatura observa-se que ao se obter um perfil somatotípico de atleta na modalidade, através das semelhanças encontradas nas categorias e diferenças entre as posições de jogo, pode-se utilizar tais dados como referência para a constante busca por talentos esportivos com características consonantes as modalidades esportivas de forma mais específica.

\section{CONCLUSÕES}

É possível concluir que a estatura associada a ectomorfia, é uma variável importante na seleção de atletas para as representações nacionais, quando a mesma não apresentou diferença entre as categorias, mostrando que desde as seleções de base tal variável faz parte dos critérios de seleção. Percebe-se ainda, que as jogadoras da posição de líbero são as de menor estatura, enquanto as centrais (ou meio de rede) são as mais altas, seguidas das demais atacantes. $\bigcirc$ somatotipo da seleção infanto-juvenil apresentou diferença significativa em relação às demais, entretanto o 
maior valor no terceiro componente, relacionado a linearidade, é característica da modalidade em todas as categorias. Observou-se também, que o somatotipo das jogadoras nas diferentes posições de jogo apresenta características próprias, nas quais as atacantes de ponta e as centrais foram classificadas como ectomorfas balanceadas, as opostas como ectomorfas-mesomorfas, as líberos como mesomorfas-ectomorfas e as levantadoras como centrais.

Anthropometry and somatotype: determinant factors in the selection of brazilian volleyball players

ABSTRACT: Objective: analyze the anthropometry and somatotype of Brazilian volleyball athletes. Methods: The sample was composed of 54 athletes convened for the national selections, 16 of the adult (25.6 \pm 5 , 3anos), 17 of juvenile (17.89 \pm 0 ,32anos) and 21 under 17 (15.86 \pm 0 , 36anos). Heath and Carter Somatotype Method was used to collect somatotype. The statistical treatment used was descriptive with values of central tendency and derivates and the inferential ANOVA one-way and post hoc of Tukey. The spatial distance between the somatotypes (DES) was used to calculate the somatotype. Results: the height variable wasn't different between the selections, however, this difference is evident between the positions of the game. The somatotype of under 17 selection was different from the others. The adult selection was classified as ectomorphic-mesomorph (2.24 / 3, 16 / 3.58), the juvenile as central (3.12 / 3.40 / 3.30) and under 17 as ecto-endomorphic (3.07 /2.25 / 3.81 ). Conclusion: the height and linearity are important variables in the selecions of athletes for high performance.

KEYWORDS: Anthropometry; somatotype; volleyball; performance.

\section{Antropometria y somatotipo: factores determinantes en la selección de atletas en el voleibol brasileño}

RESUMEN: Objetivo: analizar la antropometría y el somatotipo de atletas del voleibol brasileño. Métodos: la muestra fue composta de 54 atletas convocadas a las selecciones nacionales, com I 6 de la adulta (25,6 \pm 5 ,3anos) I 7 de la juvenil $(17,89 \pm 0,32$ anos) y 2 I de

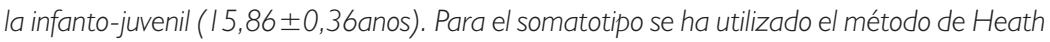
y Carter. El análisis estadístico utilizado fue el de los valores descriptivos de tendencia central y de sus derivados, inferencial y ANOVA one-way y post hoc de Tukey. Para el somatotipo se utiliza para calcular la distancia espacial entre el somatotipo (DES). Resultados: la variable de la altura no es significativamente diferente entre los equipos, sólo entre las posiciones del juego. El somatotipo de la selección infanto-juvenil fue distinto a las demás selecciones. La selecione adulta se clasificó como ectomorfo-mesomorfo $(2,24 / 3,16 / 3,58)$, juvenil como central $(3,12 / 3,40 / 3,30)$ e infanto-juvenil como ecto-endomorfico $(3,07 / 2,25 / 3,81)$. Conclusíon: La estatura y linearidade son variables importantes en la selección de atletas para el alto rendimiento.

PALABRAS CLAVES: Antropometría; somatotipo; voleibol; rendimiento. 


\section{REFERÊNCIAS}

BANDYOPADHYAY. A. Anthropometry and body composition in soccer and volleyball players in West Bengal, Índia. J Physl Anthropology. Tokyo, v. 26, p. 501-505, jun., 2007.

BAYIOS, I. A. et al. Antropometric, body composition and somatotype differences of Greek elite female basketball, volleyball and handball players. J Sports Med Phys Fitness, Torino, v. 46, n. 2, p. 27I-280, jun., 2006.

BELLENDIER, J. Una visión analítico-descriptiva del Mundial de Voleibol Argentina 2002. Revista digital, Buenos Aires, año 9, n. 60, 32-36, maio 2003.

CABRAL, S. A. T. C.; REGO, S. A. S. J.; FERNANDES FILHO, J. Características dermatoglificas, somatotipicas e das qualidades físicas da seleção infanto-juvenil de voleibol do estado do Rio Grande do Norte. Fiep Bulletin, Foz do Iguaçu, v. 74, p. 695-699, jan. 2004.

CABRAL, S. A. T. C.; BARBOSA F. P.; CABRAL, B.G.A.T.C. Correlation between dermatoghyphic characteristics and explosive force in female under- 17 volleyball athletes. Fiep Bulletin, Foz do Iguaçu, v. 75, p. 26-28, jan. 2005.

CABRAL, B. G. A. T. C. et al. Efeito discriminante da morfologia e alcance de ataque no nível de desempenho em voleibolistas. Rev Brás Cineantropom Desempenho Hum, Florianópolis, v.13, n. 3, p. 223-229, mar. 2011 .

CARTER, J. E. L., HEATH, B.H. Somatotyping: Development and applications. Cambridge: Cambridge University Press, 1990.

CBV. Confederação Brasileira de Voleibol. Disponível em: <http//www.cbv.com.br> . Acesso em: 15 maio 2010.

DUNCAN, M. J.; WOODIFIELD, H.; AL-NAKEEB. Y. Anthropometric and physiological characteristics of junior elite volleyball players. Br J Sports Med, London, v.40, n.7, p.64965I, jul. 2006.

FIVB. Federação Internacional de Voleibol. Disponível em: < http://www.fivb.org/ENNolleyball/Competitions/Youth/Women/2005/Index.asp>. Acesso em: 22 setembro 2010.

FLORES, A. A. A. et al. Anthropometric Profile of Professional Volleyball Sudamerican Players. Int J Morphol, Temuco, v.27, n. I, p. 53-57, mar. 2009.

FRITZSCHE, J.; RASCHKA, C. Sports anthropological investigations on somatotypology of elite karateka. Anthropol Anz, German, v. 65, n. 3, p.: 317-329, sep. 2007.

GABBETT, T.; GEORGIEFF, B. Physiological and anthropometric characteristics of Australian junior national, state, and novice volleyball players. J Strenght Cond Res, Lincoln, v. 2 I , n. 3 , p. 902-908, aug. 2007. 
GUALDI-RUSSO, E.; ZACCAGNI, L. Somatotype, role and performance in elite volleyball players. J Sports Med Phys Fitness, Torino, v. 4I, n. 2, p. 256-262, jun. 2001.

HEATH, B. H., CARTER, J. E. L. A modified somatotype method. Am J Phys Anthropol, Hoboken, v. 27, p. 57-74, 1967.

MALINA, R. M.; BOUCHARD, C.; BAR-OR, O. Growth, maturation and physical activity. $2^{\text {nd }}$ edition. Champaign, IL: Human Kinetics, 2004.

MALOUSARIS, G .G. et al. Somatotype, size and composition of competitive female volleyball players. J Sci Méd Sport, Ankara, v. I I , p. 337-344, aug. 2008.

MARFELL-JONES, M. et al. International Standards for Anthropometric Assessment. International Society for the Advancement of Kinanthropometry-ISAK, Potchesfstroom: Isak, 2006. I45p.

MARQUES, M. C. et al. Physical Fitness Qualities of Professional Volleyball Players: Determination of Positional Differences. J Strength Cond Res, Lincoln, v. 23, n. 4, p. I I06-I I I I, 2009.

MASSA, M. et al. Somatotipo de atletas de voleibol feminino nas diferentes categorias competitivas. Anais do XXIII Simpósio Internacional de Ciências do Esporte, São Paulo, p. I 45, 2000.

NORTON, K.; OLDS, T. Morphologia evolution of athletes over the century: causes and consequences. J Sports Med, Stuttgart v. 3 I, n. I I, p. 763-783, fev. 2001.

OKAZAKI, F. H. A. et al. The Relative Age Effect Among Female Brazilian Youth Volleyball Players. Res Q Exerc Sport, Washington, v. 82, n. I, p. I35-139, mar. 201 I.

QUINTAL, A. J. et al. Aspectos morfológicos e funcionais do atleta infanto-juvenil madeirense: Estudo em praticantes de andebol, basquetebol, futebol e voleibol. In: QUINTAL, A. et al. (Ed.). O atleta infanto-juvenil madeirense. Viseu: Tipografia Guerra, 2007. p. 25 I-355.

RASCHKA, C.; WOLTHAUSEN, C. Comparison of somatotype differences of soccer and handball players based on the methods of German and Anglo-American schools of constitutional biology. Anthropol Anz, German, v. 65, n. 3, p. 303-316, sep. 2007.

SÁNCHEZ-MUNOZ, C.; SANZ, D.; ZABALA, M. Anthropometric characteristics, body composition and somatotype of elite junior tennis players. Br J Sports Med., London, v.4I, n. II, p. 793-799, nov. 2007.

STAMM, R. et al. Dependence of Young female volleyballers performance on their body build, physical abilities, and psycho-physiological properties. J Sports Med Phys Fitness, Torino, v.43, n.3, p. 29I-299, sep. 2003.

VEIGA, V. W. M. Maturação e performance de jovem andebolista. 2009. I03f. Dissertação (Mestrado em treino do jovem atleta) - Universidade Técnica de Lisboa-Faculdade de Motricidade Humana, Lisboa, Portugal, 2009. 
VIVOLO, M. A.; CALDEIRA, S.; MATSUDO, V. K. R. Estudo antropométrico da equipe nacional de voleibol feminino do Japão segundo o método do somatotipo de Heath-Carter. Revista Volleyball. Rio de Janeiro: Palestra edições. Ano I, v. 2, p. 15-20, mar. 1980.

Recebido: 18 out. 2010 Aprovado: 27 jun. 2011

Endereço para correspondência Breno Guilherme de A.T. Cabral Av. Amintas Barros, 3675, Apt. I60 I Lagoa Nova, Natal/RN, Brasil

CEP: $59075-250$ 